Pacific Journal of Mathematics

ON THE MAXIMAL NUMBER OF LINEARLY INDEPENDENT

BAL QUADRATIC 


\section{ON THE MAXIMAL NUMBER OF LINEARLY INDEPENDENT REAL VECTORS ANNIHILATED SIMULTANEOUSLY BY TWO REAL QUADRATIC FORMS}

\section{FRANK UHLIG}

For a nonsingular pair of real symmetric (r.s.) matrices $S$ and $T$ the maximal number $m$ of lin. ind. vectors simultaneously annihilated by the associated quadratic forms is computed as a function of the real Jordan normal form of $S^{-1} T$. Conversely one can deduce which real Jordan normal form $S^{-1} T$ must have, if a specific $m$ is the maximal number of such vectors. Furthermore, two new conditions are found that assure $S$ and $T$ to be simultaneously diagonalizable by a real congruence transformation.

First we introduce the notions of Jordan blocks, real Jordan normal form and the canonical pair form for pairs of r.s. matrices.

Definition 0.1. A square matrix of the form

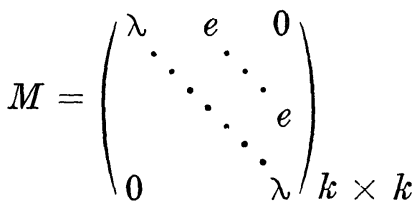

is called a Jordan block of type (A), if for $k \geqq 2$ we have $\lambda \in R$ and $e=1$, while for $k=1$ we have $M=(\lambda)$ with $\lambda \in R$. Such a matrix $M$ is called a Jordan block of type (B), if for $k \geqq 4$ we have $\lambda=$ $\left(\begin{array}{rr}a-b \\ b\end{array}\right), a, b \in R, b \neq 0$ and $e=\left(\begin{array}{ll}1 & 0 \\ 0 & 1\end{array}\right)$, while for $k=2$ we have $M=$ $\left(\begin{array}{r}a-b \\ b\end{array}\right)$ with $a, b \in \boldsymbol{R}, b \neq 0$. Jordan blocks will also be denoted by $J(\lambda, k)$ and $J(a, b, k)$, respectively.

Now we can state the real Jordan normal form theorem (see, e.g., Kowalski [2], p. 248).

TheOREM 0.1. Every real square matrix $A$ is similar over the reals to a matrix $J=\operatorname{diag}\left(A_{1}, \cdots, A_{l}\right)$, in which each square block $A_{j}$ corresponds to an eigenvalue $\lambda_{j}$ of $A$. If this eigenvalue $\lambda_{j}$ is real, the associated $A_{j}$ is a Jordan block of type (A); if $\lambda_{j}=a+b i \notin \boldsymbol{R}$, then $A_{j}$ is a Jordan block of type (B). This $J$ is called the real Jordan normal form of $A$. It is uniquely determined by $A$, except for the order of its Jordan blocks. 
The final result to be quoted will be the canonical pair form theorem for nonsingular pairs of r.s. matrices, that is pairs $S$ and $T$ where $S$ is nonsingular.

TheOREm 0.2. Let $S$ and $T$ be a nonsingular pair of r.s. matrices. Let $S^{-1} T$ have real Jordan normal form $\operatorname{diag}\left(J_{1}, \cdots, J_{r}, J_{r+1}, \cdots, J_{m}\right)$, where $J_{1}, \cdots, J_{r}$ are Jordan blocks of type (A) corresponding to real eigenvalues of $S^{-1} T$ and $J_{r+1}, \cdots, J_{m}$ are Jordan blocks of type (B) for pairs of complex conjugate roots of $S^{-1} T$.

Then $S$ and $T$ are simultaneously congruent by a real congruence transformation to

and

$$
\operatorname{diag}\left(\varepsilon_{1} E_{1}, \cdots, \varepsilon_{r} E_{r}, E_{r+1}, \cdots, E_{m}\right)
$$

$$
\operatorname{diag}\left(\varepsilon_{1} E_{1} J_{1}, \cdots, \varepsilon_{r} E_{r} J_{r}, E_{r+1} J_{r+1}, \cdots, E_{m} J_{m}\right)
$$

respectively, where $\varepsilon_{i}= \pm 1$ and $E_{i}$ denotes the square matrix $\left(\begin{array}{cc}0 & 1 \\ . & \cdot \\ 1 & 0\end{array}\right)$ of the same size as $J_{i}$ for $i=1, \cdots, m$.

Canonical forms for a pair of r.s. matrices go back to Weierstra $\beta$ and Kronecker. A list of references can be found in Uhlig [3], Theorem 0.4 .

Notation. For $S$ symmetric we define $Q_{S}=\left\{x \in \boldsymbol{R}^{n} \mid x^{\prime} S x=0\right\}$.

We will now state the main theorems that relate $m=\max \{k \mid$ there exist $k$ lin. ind. vectors in $Q_{S} \cap Q_{T}$ \} to the real Jordan normal form of $S^{-1} T$.

Theorem 1. Let $S$ and $T$ be a nonsingular pair of r.s. $n \times n$ matrices. Let $J$ be the real Jordan normal form of $S^{-1} T$. If

(i) $J$ contains a Jordan block of dimension greater than 3, or

(ii) $J$ contains two Jordan blocks of dimension 3 each, or

(iii) $J$ contains one Jordan block of dimension 3 and one of dimension 2, or

(iv) $n>3$ and $J$ contains a Jordan block of dimension 3 and 1-dimensional blocks else, but not all eigenvalues of $S^{-1} T$ are the same, or

( v ) $J$ contains two 2-dimensional Jordan blocks which correspond to different eigenvalues of $S^{-1} T$ if both blocks are of type (A), $\cdots$, then $Q_{S} \cap Q_{T}$ contains $n$ linearly independent vectors.

Theorem 2. Let $S$ and $T$ be a nonsingular pair of r.s. matrices of dimension $n$. Let $J$ be the real Jordan normal form of $S^{-1} T$. If 
(vi) $n>3, J$ contains one 3-dimensional Jordan block, linear blocks else and all eigenvalues of $S^{-1} T$ are the same while inertia $S \neq(n-1,1,0),(1, n-1,0)$; or

(vii) $n>3$ and $J$ contains $k \geqq 1$ identical 2-dimensional Jordan blocks $J(\lambda, 2)$ of type (A), linear blocks else for eigenvalues $\mu_{i}(i=$ $2 k+1, \cdots, n)$ and the set

$$
\left\{\varepsilon_{1}, \cdots, \varepsilon_{k}, \varepsilon_{i}\left(\mu_{i}-\lambda\right) \mid i>2 k\right\}
$$

contains positive as well as negative numbers, where the $\varepsilon_{j}= \pm 1$ are the constants in the canonical pair form of $S$ and $T$ (see Theorem 0.2), or

(viii) $n>3, J$ contains one 2-dimensional block $J(a, b, 2)$ of type (B) and linear blocks else for eigenvalues $\mu_{i}$, where not all $\mu_{i}$ are the same or ${ }^{1}$ inertia $S \neq(n-1,1,0),(1, n-1,0)$,

then $Q_{S} \cap Q_{T}$ contains $n$ linearly independent vectors. If

(vi) (a) condition (vi) holds, except that inertia $S=(n-1,1,0)$ or $(1, n-1,0)$, or

(viii) (a) condition (viii) holds, except that all real eigenvalues $\mu_{i}$ as defined in (viii) are the same and inertia $S=(n-1,1,0)$ or $(1, n-$ $1,0)$. Then $Q_{S} \cap Q_{T}$ contains a maximum of $n-1$ lin. indep. vectors only. If

(vii) (a) condition (vii) holds except that the set $\left\{\varepsilon_{1}, \cdots, \varepsilon_{k}, \varepsilon_{i}\left(\mu_{i}-\right.\right.$ $\lambda) \mid i>2 k\}$ as defined in (vii) contains $r \geqq 0$ zeroes $\mu_{2 k+1}-\lambda=\cdots=$ $\mu_{2 k+r}-\lambda=0$ and only positive or only negative numbers else, and $\varepsilon_{2 k+1}=\cdots=\varepsilon_{2 k+r}$, then $Q_{S} \cap Q_{T}$ contains a maximum number of $k$ lin. ind. vectors. If

(vii) (b) condition (vii) (a) holds except that not all $\varepsilon_{i}$ are the same for $2 k+1 \leqq i \leqq 2 k+r$, then $Q_{S} \cap Q_{T}$ contains a maximum of $k+r$ lin. ind. vectors. If

(ix) $n>1$ and $S$ and $T$ can be simultaneously diagonalized by a real congruence transformation, then the maximal number $k$ of lin. ind. vectors in $Q_{S} \cap Q_{T}$ can be $k=0,2, \cdots, n$ depending on $S$ and $T$.

Theorem 3 will treat $n$-dimensional r.s. matrix pairs for $n \leqq 3$ : The following lemma is useful for the proofs of Theorems 1 and 2.

LEMma 1. Let $S$ and $T$ be real symmetric matrices and $A$ be a real nonsingular matrix.

Then

$\max \left\{k \mid\right.$ there exist $k$ lin. ind. vectors in $\left.Q_{S} \cap Q_{T}\right\}$ $=\max \left\{k \mid\right.$ there exist $k$ lin. ind. vectors in $\left.Q_{A^{\prime} S A} \cap Q_{A^{\prime} T_{A}}\right\}$. 
This is obvious if one observes that $Q_{A^{\prime} S A}=A^{-1}\left(Q_{S}\right)$ for nonsingular $A$.

Proof. (Theorem 1) In view of the above lemma we may assume without loss of generality that $S$ and $T$ are in canonical pair form:

$$
\begin{aligned}
& S=\operatorname{diag}\left( \pm E_{1}, \cdots, \pm E_{r}, E_{r+1}, \cdots, E_{m}\right), \\
& T=\operatorname{diag}\left( \pm E_{1} J_{1}, \cdots, \pm E_{r} J_{r}, E_{r+1} J_{r+1}, \cdots, E_{m} J_{m}\right),
\end{aligned}
$$

where $J_{1}, \cdots, J_{r}$ are Jordan blocks of type (A) and $J_{r+1}, \cdots, J_{m}$ are of type (B).

Having $S$ and $T$ in this form is very advantageous. For then we have $\left(e_{i}^{\prime} S e_{i}\right)^{2}+\left(e_{i}^{\prime} T e_{i}\right)^{2} \neq 0$ for at most $r+2(m-r)$ unit vectors $e_{i}$. The reason is as follows:

For the Jordan block $J_{1}=J(\lambda, k)$ of type (A) we have: if $k$ is even:

$$
e_{k / 2+1}^{\prime} E e_{k / 2+1}=0
$$

and

$$
e_{k / 2+1}^{\prime} E J(\lambda, k) e_{k / 2+1}=\lambda,
$$

while for all other $i \leqq k: e_{i}^{\prime} E e_{i}=e_{i}^{\prime} E J(\lambda, k) e_{i}=0_{j}$, if $k$ is odd:

$$
e_{k+1 / 2}^{\prime} E e_{k+1 / 2}=1
$$

and

$$
e_{k+1 / 2}^{\prime} E J(\lambda, k) e_{k+1 / 2}=\lambda,
$$

while for all other $i \leqq k: e_{i}^{\prime} E e_{i}=e_{i}^{\prime} E J(\lambda, k) e_{i}=0$. For the Jordan block $J_{1}=J(a, b, k)(b \neq 0)$ of type (B) we have: if $k$ is divisible by $4: e_{i}^{\prime} E e_{i}=$ $e_{i}^{\prime} E J(\alpha, b, k) e_{i}=0$ for all $i \leqq k$; while for a $k$ not divisible by 4 we have

$$
\begin{aligned}
& e_{k / 2}^{\prime} E e_{k / 2}=0, e_{k / 2}^{\prime} E J(a, b, k) e_{k / 2}=b ; e_{k / 2+1}^{\prime} E e_{k / 2+1}=0, \\
& e_{k / 2+1}^{\prime} E J(a, b, k) e_{k / 2+1}=-b \text { and } e_{i}^{\prime} E e_{i}=e_{i}^{\prime} E J(a, b, k) e_{i}=0
\end{aligned}
$$

for all other $i \leqq k$.

The same argument holds for each of the Jordan blocks. So there are at most $r+2(m-r)$ unit vectors not simultaneously annihilated by the two quadratic forms $x^{\prime} S x$ and $x^{\prime} T x$ if $S$ and $T$ are in canonical pair form. For all $i$ such that $e_{i} \notin Q_{S} \cap Q_{T}$ we will exhibit lin. ind. vectors $y_{i} \in Q_{S} \cap Q_{T}$ that have a nonzero $i$ th component and hence are also lin. ind. of all $e_{i}$ with $e_{i} \in Q_{S} \cap Q_{T}$. Then Theorem 1 is proved: There are $n$ lin. ind. vectors in $Q_{S} \cap Q_{T}$.

The remainder of this proof will consist of finding these vectors $y_{i}$, one for each Jordan block of type (A), two for each Jordan block of type (B) of dimension not divisible by 4 in each of the cases (i), $\cdots$, (v).

From now on we will in general assume that the Jordan blocks 
of $S^{-1} T$ mentioned in (i), $\cdots,(\mathrm{v})$ appear in the first diagonal positions. Before starting on the individual cases we express the quadratic forms corresponding to $S$ and $T$ by only singling out the first block here: If a Jordan block $J(\lambda, k)=J_{1}$ of type (A) appears first, let us look at the two quadratic forms $F(x)=x^{\prime} S x$ and $G(x)=x^{\prime} T x$ :

For

$$
S=\operatorname{diag}\left( \pm E_{1}, \cdots, \pm E_{m}\right) \text { and } x=\left(x_{1}, \cdots, x_{n}\right)
$$

we have

$$
F(x)= \pm h(x)+f(x)
$$

where

$$
h(x)=x^{\prime} \operatorname{diag}\left(E_{1}, 0, \cdots, 0\right) x=\sum_{i+j=k+1} x_{i} x_{j}
$$

and

$$
f(x)=x^{\prime} \operatorname{diag}\left(0, \pm E_{2}, \cdots, \pm E_{m}\right) x
$$

is a quadratic form involving $x_{k+1}, \cdots, x_{n}$ only.

For

$$
T=\operatorname{diag}\left( \pm E_{1} J_{1}, \cdots, \pm E_{m} J_{m}\right)
$$

we have

$$
G(x)= \pm(\lambda h(x)+e(x))+g(x)
$$

where $h$ is as above,

$$
e(x)=\sum_{i+j=k+2} x_{i} x_{j} \text { for } i, j \leqq k
$$

and

$$
g(x)=x^{\prime} \operatorname{diag}\left(0, \pm E_{2} J_{2}, \cdots, \pm E_{m} J_{m}\right) x
$$

involves $x_{k+1}, \cdots, x_{n}$ only.

Now $F(x)=0$ iff $f(x)=\mp h(x)$. And by definition $x \in Q_{S} \cap Q_{T}$ iff $F(x)=G(x)=0$ hence iff

$$
\pm e(x)+g(x)-\lambda f(x)=0 \text { and } F(x)=0 .
$$

If a Jordan block $J(a, b, k)=J_{1}(b \neq 0)$ of type (B) appears first in $S^{-1} T$, then we define $F(x)=x^{\prime} S x=h(x)+f(x)$ with $h$ and $f$ as above and $G(x)=x^{\prime} T x=a h(x)+b t(x)+u(x)+g(x)$, where $h$ and $g$ are as above and

$$
u(x)=\sum_{\substack{i+j=k+3 \\ i, j \leqq k}} x_{i} x_{j}, \quad \text { while } t(x)=\sum_{\substack{i+j=k \\ i, j \text { odd }}} x_{i} x_{j}-\sum_{\substack{i+j=k+2 \\ i, j=v+2 \\ i, j \leqq k}} x_{i} x_{j} .
$$

Thus in this case $x \in Q_{S} \cap Q_{T}$ iff $F(x)=G(x)=0$, hence iff 


$$
b t(x)+u(x)+g(x)-a f(x)=0 \text { and } \quad F(x)=0 .
$$

(i ): Assume (i) holds with a Jordan block $J(\lambda, k)$ of type (A) for $k \geqq 4$. Then from above there is an $i, 2<i<k$, such that $e_{i} \notin$ $Q_{S} \cap Q_{T}$. For this index $i$ we define $\alpha_{i}, \beta_{i} \in \boldsymbol{R}$ and $y_{i}=\alpha_{i} e_{1}+\beta_{i} e_{2}+$ $e_{i}+e_{k}$ such that (1) holds: $\pm e\left(y_{i}\right)+g\left(y_{i}\right)-\lambda f\left(y_{i}\right)= \pm\left(2 \beta_{i}+e\left(e_{i}\right)\right)=0$ determines $\beta_{i}$ and $F\left(y_{i}\right)=0$ determines $\alpha_{i}$.

For $i>k$ such that $e_{i} \notin Q_{S} \cap Q_{T}$ and $g\left(e_{i}\right)-\lambda f\left(e_{i}\right)=0$, we define the vector $y_{i}=\alpha_{i} e_{1}+e_{k}+e_{i}$, where $\alpha_{i}$ is such that $F\left(y_{i}\right)=0$. In the case that $g\left(e_{i}\right)-\lambda f\left(e_{i}\right) \neq 0$ we define $y_{i}=\alpha_{i} e_{1}+\beta_{i} e_{2}+e_{k}+e_{i}$, where $\alpha_{i}, \beta_{i} \in \boldsymbol{R}$ are such that (1) holds: $+2 \beta_{i}+g\left(e_{i}\right)-\lambda f\left(e_{i}\right)=0$ defines $\beta_{i}$ and $F\left(y_{i}\right)=0$ defines $\alpha_{i}$.

Next assume (i) holds for a Jordan block $J(a, b, k)$ of type (B) for $k=2 l \geqq 4$.

First assume $k=2 l$ is divisible by 4 . Then $e_{i} \notin Q_{S} \cap Q_{T}$ implies $i>k$ as pointed out above. For such an $i$ define $y_{i}=\alpha_{i} e_{l-1}+\beta_{i} e_{l}+$ $e_{l+1}+e_{i}$ where $\alpha_{i}, \beta_{i} \in \boldsymbol{R}$ are such that (2) holds. When checking (2), note that $l$ is even, if $k$ is divisible by $4.2 b \alpha_{i}+g\left(e_{i}\right)-a f\left(e_{i}\right)=0$ defines $\alpha_{i}$ and $2 \beta_{i}+h\left(e_{i}\right)=0$ defines $\beta_{i}$.

Now assume $k=2 l$ is not divisible by 4 . Then $l$ is odd and we know that both $e_{l}, e_{l+1} \notin Q_{S} \cap Q_{T}$ from the above. If we define

$$
y_{l}=e_{l}-\frac{b}{2} e_{l+3} \quad \text { and } \quad y_{l+1}=e_{l+1}+\frac{b}{2} e_{l+2},
$$

then (2) holds for these two vectors. For $i>k$ such that $e_{i} \notin Q_{S} \cap Q_{T}$ we define as before for the real case $y_{i}=\alpha_{i} e_{1}+e_{k}+e_{i}$ if $g\left(e_{i}\right)-\lambda f\left(e_{i}\right)=$ 0 and $y_{i}=\alpha_{i} e_{1}+\beta_{i} e_{2}+e_{k}+e_{i}$ otherwise. This proves (i) of Theorem 1.

(ii): Assume $J$ contains two Jordan block of dimensions 3 each. Then these must be Jordan blocks of type (A); $J(\lambda, 3)$ and $J(\mu, 3)$ for $\lambda, \mu \in \boldsymbol{R}$. Define for $x=\left(x_{1}, \cdots, x_{n}\right)$ :

$$
F(x)=x^{\prime} S x=\varepsilon\left(2 x_{1} x_{3}+x_{2}^{2}\right)+\delta\left(2 x_{4} x_{6}+x_{5}^{2}\right)+f(x)
$$

and

$$
\begin{aligned}
G(x)= & x^{\prime} T x=\varepsilon\left(\lambda\left(2 x_{1} x_{3}+x_{2}^{2}\right)+2 x_{2} x_{3}\right) \\
& +\delta\left(\mu\left(x_{4} x_{6}+x_{5}^{2}\right)+2 x_{5} x_{6}\right)+g(x),
\end{aligned}
$$

where $f$ and $g$ are quadratic forms not involving $x_{1}, \cdots, x_{6}$ and $\varepsilon, \delta= \pm 1$, independently from the canonical pair form.

Now $e_{2}, e_{5} \notin Q_{S} \cap Q_{T}$. And for these indices define the vectors $y_{2}=-1 / 2 e_{1}-\delta \varepsilon e_{2}+e_{3}-1 / 2 e_{4}+e_{5}+e_{6}$ and $y_{5}=-1 / 2 e_{1}-\delta \varepsilon e_{2}+e_{3}+$ $1 / 2 e_{4}-e_{5}-e_{6}$. They are lin. ind. and satisfy $F\left(y_{i}\right)=G\left(y_{i}\right)=0$ in (3). For $i>6$ such that $e_{i} \notin Q_{S} \cap Q_{T}$ we define 


$$
y_{i}=\alpha_{i} e_{1}+\beta_{i} e_{2}+e_{3}+e_{i},
$$

where $\beta_{i}$ is chosen such that

$$
2 \varepsilon \beta_{i}+g\left(e_{i}\right)-\lambda f\left(e_{i}\right)=0,
$$

and $\alpha_{i}$ is such that

$$
F\left(y_{i}\right)=\varepsilon\left(2 \alpha_{i}+\beta_{i}^{2}\right)+f\left(e_{i}\right)=0 .
$$

Then $G\left(y_{i}\right)=0$, too.

This completes (ii).

(iii): Here again the 3-dimensional Jordan block has to be of type (A): $J(\lambda, 3)$, while the 2-dimensional block can be of either type. Let for $x=\left(x_{1}, \cdots, x_{n}\right)$,

$$
F(x)=x^{\prime} S x=\varepsilon\left(2 x_{1} x_{3}+x_{2}^{2}\right)+\delta\left(2 x_{4} x_{5}\right)+f(x)
$$

and

$$
\begin{aligned}
& G(x)=x^{\prime} T x=\varepsilon\left(\lambda\left(2 x_{1} x_{3}+x_{2}^{2}\right)+2 x_{2} x_{3}\right) \\
& +\delta\left\{\begin{array}{l}
\left(2 \mu x_{4} x_{5}+x_{5}^{2}\right) \\
\left(2 a x_{4} x_{5}+b\left(x_{4}^{2}-x_{5}^{2}\right)\right)
\end{array}\right\}+g(x) \text { in case of (A) }
\end{aligned}
$$

where $\delta, \varepsilon= \pm 1$ from the canonical pair form and $f$ and $g$ do not involve the first five components. If the 2-dimensional Jordan block in question is of type (A), then for $i \leqq 5$ we have $e_{i} \notin Q_{S} \cap Q_{T}$ exactly for $i=2,5$, while for a Jordan block of type (B) those indices are $i=2,4,5$.

In case of $(A)$ define

$$
\begin{aligned}
& y_{2}=\delta \varepsilon e_{1}+e_{2}-\frac{\delta \varepsilon}{2} e_{3}+e_{5} \\
& y_{5}=\delta \varepsilon e_{1}+e_{2}-\frac{\delta \varepsilon}{2} e_{3}-e_{5}
\end{aligned}
$$

and one has $y_{2}, y_{5} \in Q_{S} \cap Q_{T}$.

In case of a 2-dimensional block $J(a, b, 2), b \neq 0$ of type (B), define

$$
\begin{aligned}
& y_{2}=-\frac{\varepsilon}{b} e_{1}+e_{2}+\frac{b}{2} \varepsilon e_{3}+e_{5} \\
& y_{4}=+\frac{\varepsilon}{b} e_{1}+e_{2}-\frac{b}{2} \varepsilon e_{3}+e_{4} \\
& y_{5}=-\frac{\varepsilon}{b} e_{1}+e_{2}+\frac{b}{2} \varepsilon e_{3}-e_{5} .
\end{aligned}
$$

Then $y_{2}, y_{4}, y_{5} \in Q_{S} \cap Q_{T}$. 
For $i>5$ such that $e_{i} \in Q_{S} \cap Q_{T}$, define $y_{i}=\alpha_{i} e_{1}+\beta_{i} e_{2}+e_{3}+e_{i}$, where $\alpha_{i}, \beta_{i} \in \boldsymbol{R}$ are such that $2 \varepsilon \beta_{i}+g\left(e_{i}\right)-\lambda f\left(e_{i}\right)=0$ and $F\left(y_{i}\right)=0$. This concludes part (iii).

(iv): Here we have $F(x)=x^{\prime} S x=\varepsilon\left(2 x_{1} x_{3}+x_{2}^{2}\right)+f(x)$ and $G(x)=$ $x^{\prime} T x=\varepsilon\left(\lambda\left(2 x_{1} x_{3}+x_{2}^{2}\right)+2 x_{2} x_{3}\right)+g(x)$ and $F(x)=G(x)=0$ iff

$$
\varepsilon 2 x_{2} x_{3}+g(x)-\lambda f(x)=0 \text { and } F(x)=0 .
$$

By assumption all but the first Jordan block $J(\lambda, 3)$ in $S^{-1} T$ are 1dimensional blocks $J\left(\mu_{i}, 1\right)$. We assume $n>3$, so there exists an $i_{0}>3$ such that $g\left(e_{i_{0}}\right)-\lambda f\left(e_{i_{0}}\right) \neq 0$, for $g\left(e_{i}\right)-\lambda f\left(e_{i}\right)= \pm\left(\mu_{i}-\lambda\right)=0$ for all $i>3$ contradicts our assumption.

Now $e_{2} \notin Q_{S} \cap Q_{T}$ and we define $y_{2}=\alpha_{2} e_{1}+\beta_{2} e_{2}+e_{3}+e_{i_{0}}$, where $\beta_{2} \neq 0$ is such that $2 \varepsilon \beta_{2}+g\left(e_{i_{0}}\right)-\lambda f\left(e_{i_{0}}\right)=0$ and $\alpha_{2}$ is such that $F\left(y_{2}\right)=0$. For all $i>3$ we have $e_{i} \notin Q_{S} \cap Q_{T}$ and we define $y_{i_{0}}=-$ $\alpha_{2}^{\prime} e_{1}-\beta_{2} e_{2}-e_{3}+e_{i_{0}}$ and $y_{i}=\alpha_{i} e_{1}+\beta_{i} e_{2}+e_{3}+e_{i}$ for all other $i>3$, where the $\alpha$ 's and $\beta$ 's are chosen such that (4) holds for all $y_{i}$. These $n$ vectors $y_{i}$ are lin. ind.

(v): Now only (v) remains to be proved. Let us first assume that the two 2-dimensional Jordan blocks in question are both of type (A): $J(\lambda, 2), J(\mu, 2)$, where by assumption $\lambda \neq \mu$. Then $F(x)=x^{\prime} S x=$ $\varepsilon 2 x_{1} x_{2}+\delta 2 x_{3} x_{4}+f(x)$ and $G(x)=x^{\prime} T x=\varepsilon\left(2 \lambda x_{1} x_{2}+x_{2}^{2}\right)+\delta\left(2 \mu x_{3} x_{4}+x_{4}^{2}\right)+$ $g(x)$ where $\varepsilon, \delta= \pm 1$ and $f$ and $g$ do not involve the first four components of $x$. Then $F(x)=G(x)=0$ is equivalent to

$$
F(x)=0 \text { and } 2 \delta(\mu-\lambda) x_{3} x_{4}+\varepsilon x_{2}^{2}+\delta x_{4}^{2}+g(x)-\lambda f(x)=0 .
$$

Now if $e_{i} \notin Q_{S} \cap Q_{T}$, then $i=2$ or $i=4$, unless $i>4$. We define

$$
\begin{aligned}
& y_{2}=\alpha e_{1}+2 e_{2}+\beta e_{3}-e_{4} \\
& y_{4}=\alpha e_{1}+2 e_{2}+\beta e_{3}+e_{4}
\end{aligned}
$$

and

$$
y_{i}=\alpha_{i} e_{1}+\gamma_{i} e_{2}+\beta_{i} e_{3}+e_{4}+e_{i}
$$

for all $i>4$ with $e_{i} \notin Q_{S} \cap Q_{T}$. Here $\gamma_{i} \neq 0$ are chosen such that $\varepsilon \gamma_{i}^{2}+\delta+g\left(e_{i}\right)-\lambda f\left(e_{i}\right) \neq 0$ while the $\alpha$ 's and $\beta$ 's are chosen such that (5) holds.

Next assume, the two 2-dimensional blocks are both of type (B): $J(a, b, 2), J(c, d, 2)$ where $b, c \neq 0$.

Then $F(x)$ is as above with $\varepsilon=\delta=1$ while

$$
G(x)=x^{\prime} T x=2 a x_{1} x_{2}+2 c x_{3} x_{4}+b\left(x_{1}^{2}-x_{2}^{2}\right)+d\left(x_{3}^{2}-x_{4}^{2}\right)+g(x),
$$

and $F(x)=G(x)=0$ is equivalent to 


$$
F(x)=0 \text { and } \begin{aligned}
2(c-a) x_{3} x_{4} & +b\left(x_{1}^{2}-x_{2}^{2}\right) \\
& +d\left(x_{3}^{2}-x_{4}^{2}\right)+g(x)-a f(x)=0 .
\end{aligned}
$$

Here we have $e_{i} \notin Q_{S} \cap Q_{T}$ for all $i \leqq 4$.

If $b d>0$ we define the following four lin. ind. vectors

$$
\begin{array}{ll}
y_{1}=\alpha e_{1}+\beta e_{4}, & y_{2}=\alpha e_{1}-\beta e_{4}, \\
y_{3}=\alpha e_{2}+\beta e_{3}, & y_{4}=\alpha e_{2}-\beta e_{3},
\end{array}
$$

where $\alpha, \beta \neq 0$ are such that $b \alpha^{2}-d \beta^{2}=0$ and thus (6) holds for all $y_{i}, i \leqq 4$.

In $b d<0$, we define $y_{i}$ as follows:

$$
\begin{array}{ll}
y_{1}=\alpha e_{1}+\beta e_{3}, & y_{2}=\alpha e_{1}-\beta e_{3}, \\
y_{3}=\alpha e_{2}+\beta e_{4}, & y_{4}=\alpha e_{2}-\beta e_{4},
\end{array}
$$

where $\alpha, \beta \neq 0$ satisfy $b \alpha^{2}+d \beta^{2}=0$ such that all four $y_{i}$ satisfy (6) again.

For indices $i>4$ such that $e_{i} \notin Q_{S} \cap Q_{T}$ we define the corresponding vector $y_{i}$ as follows:

If $f\left(e_{i}\right)=0$ and $b d>0$, let $y_{i}=\alpha_{i} e_{1}+\beta_{i} e_{4}+e_{i}$, where $\alpha_{i}, \beta_{i}$ are chosen such that $b \alpha_{i}^{2}-d \beta_{i}^{2}=-g\left(e_{i}\right)$. If $f\left(e_{i}\right)=0$ and $b d<0$, let $y_{i}=$ $\alpha_{i} e_{1}+\beta_{i} e_{3}+e_{i}$, where $\alpha_{i}, \beta_{i} \in \boldsymbol{R}$ such that $b \alpha_{i}^{2}+d \beta_{i}^{2}=-g\left(e_{i}\right)$. If $f\left(e_{i}\right) \neq 0$ and $g\left(e_{i}\right)-a f\left(e_{i}\right)=0$, then let $y_{i}=\alpha_{i} e_{1}+\beta_{i} e_{2}+e_{i}$ where $\left|\alpha_{i}\right|=\left|\beta_{i}\right|$ such that $y_{i}$ satisfies (6). If $f\left(e_{i}\right) \neq 0$ and $\left(g\left(e_{i}\right)-a f\left(e_{i}\right)\right) d>$ 0 , let $y_{i}=\alpha_{i} e_{1}+\beta_{i} e_{2}+\gamma_{i} e_{4}+e_{i}$, where $\left|\alpha_{i}\right|=\left|\beta_{i}\right|$ and $\gamma_{i}$ are chosen such that (6) holds. If $f\left(e_{i}\right) \neq 0$ and $\left(g\left(e_{i}\right)-\alpha f\left(e_{i}\right)\right) d<0$, let $y_{i}=\alpha_{i} e_{1}+$ $\beta_{i} e_{2}+\gamma_{i} e_{3}+e_{i}$, with $\alpha_{i}, \beta_{i}, \gamma_{i}$ chosen to satisfy (6).

Finally we prove (v) for a Jordan block of type (A) and one of type (B): $J(\lambda, 2), J(a, b, 2)$. Then $F(x)$ is as above with $\varepsilon= \pm 1$, $\delta=1$ while $G(x)=x^{\prime} T x=\varepsilon\left(2 \lambda x_{1} x_{2}+x_{2}^{2}\right)+2 a x_{3} x_{4}+b\left(x_{3}^{2}-x_{4}^{2}\right)+g(x)$ where $g(x)$ does not involve $x_{1}, \cdots, x_{4}$. And $F(x)=G(x)=0$ is equivalent to:

$$
\begin{aligned}
F(x)=0 \text { and } \quad 2(a-\varepsilon \lambda) x_{3} x_{4} & +\varepsilon x_{2}^{2}+b\left(x_{3}^{2}-x_{4}^{2}\right) \\
& +g(x)-\lambda f(x)=0 .
\end{aligned}
$$

If $e_{i} \notin Q_{S} \cap Q_{T}$, then $i=2,3,4$ or $i>4$. We define $y_{2}$ and $y_{3}$ first:

$$
\begin{aligned}
& y_{2}=e_{2}+\beta e_{3}+\gamma e_{4} \\
& y_{3}=e_{2}-\beta e_{3}-\gamma e_{4},
\end{aligned}
$$

where $\beta=\sqrt{-\varepsilon / b}, \gamma=0$, if $\varepsilon \cdot b<0$ and $\beta=0, \gamma=\sqrt{\varepsilon / b}$, if $\varepsilon b>0$. Then $e_{1}, y_{2}$, and $y_{3}$ are all lin. ind. and satisfy (7).

If $y_{4}$ has all of its first four components nonzero it will be lin. ind. of $e_{1}, y_{2}, y_{3}$ and all $e_{i}$ for $i>4$. So let $y_{4}=\alpha e_{1}+\beta e_{2}+\gamma e_{3}+$ 
$\eta e_{4}$ where $\alpha, \beta, \gamma, \eta$ are chosen as follows:

If $a-\varepsilon \lambda=0$, take $\gamma=1, \eta=2, \beta=\sqrt{3 b \varepsilon}$, if $\varepsilon b>0$ and $\alpha \neq 0$ such that $F\left(y_{4}\right)=0$; but if $\varepsilon b<0$, choose $\gamma=2, \eta=1, \beta=\sqrt{-3 b \varepsilon}$ and $\alpha$ as above, and $y_{4}$ satisfies (7).

If $a-\varepsilon \lambda \neq 0$, choose $\eta \neq 0, \gamma=1 / \eta$ such that

$$
2(a-\varepsilon \lambda)+b\left(\frac{1}{\eta^{2}}-\eta^{2}\right)<0 \quad \text { if } \varepsilon=1
$$

and

$$
2(a-\varepsilon \lambda)+b\left(\frac{1}{\eta^{2}}-\eta^{2}\right)>0 \quad \text { if } \varepsilon=-1 .
$$

Then choose $\beta \neq 0$ such that the second equation in (7) holds and after letting $\alpha=-\varepsilon / \beta$ the vector $y_{4}$ again satisfies (7). For $i>4$ define $y_{i}=\alpha e_{1}+e_{2}+\beta e_{3}+\gamma e_{4}+e_{i}$ where $\alpha \in \boldsymbol{R}$ and either $\beta=0$ or $\gamma=0$ as before in such a way that (7) holds for each $y_{i}, i>4$. This completes the proof of Theorem 1 .

We now go on to prove Theorem 2

Proof. (Theorem 2) We use the notation of the previous proof

(vi), (vi)(a): Let (vi) or (vi)(a) hold. Then the 3 dimensional Jordan block is of type (A): $J(\lambda, 3)$. And we have with $x=\left(x_{1}\right.$, $\left.\cdots, x_{n}\right)$

$$
F(x)=x^{\prime} S x=\varepsilon_{1}\left(2 x_{1} x_{3}+x_{2}^{2}\right)+\sum_{i=4}^{n} \varepsilon_{i} x_{i}^{2}
$$

and

$$
G(x)=x^{\prime} T x=\varepsilon_{1}\left(\lambda\left(2 x_{1} x_{3}+x_{2}^{2}\right)+2 x_{2} x_{3}\right)+\sum_{i=4}^{n} \varepsilon_{i} \lambda x_{i}^{2},
$$

where $\varepsilon_{i}= \pm 1$. Hence $F(x)=G(x)=0$ is equivalent to $F(x)=0$ and $x_{2} x_{3}=0$.

If all $\varepsilon_{i}$ are the same, then, since inertia $E_{3 \times 3}=(2,1,0)$, we have inertia $S=(n-1,1,0)$ or $(1, n-1,0)$ and (vi)(a) would hold. But let us first assume (vi) holds. Then for some $l \geqq 4$ we must have $\varepsilon_{1} \cdot \varepsilon_{l}<0$. Clearly $e_{1}, e_{3} \in Q_{S} \cap Q_{T}$ and for the other indices we define:

$$
\begin{aligned}
& y_{2}=e_{2}+e_{l} \\
& y_{i}=\frac{\varepsilon_{1} \varepsilon_{i}}{2} e_{1}-e_{3}+e_{i} \quad \text { for } i \geqq 4 .
\end{aligned}
$$


Then $e_{1}, e_{3}, y_{2}$ and $y_{i}(i \geqq 4)$ are in $Q_{S} \cap Q_{T}$ and are lin. ind.

If (vi) (a) holds, then $F(x)=0$ and $x_{2} x_{3}=0$ implies $x_{2}=0$, such that we cannot find a vector $y_{2} \in Q_{S} \cap Q_{T}$ with a nonzero second component. Hence $Q_{S} \cap Q_{T}$ contains at most $(n-1)$ lin. ind. vectors. But $e_{1}, e_{3}, y_{i}(i \geqq 4)$ defined above are linearly independent and belong to $Q_{S} \cap Q_{T}$. This proves (vi) and (vi)(a).

(vii), (vii)(a), (vii)(b): We define

$$
F(x)=x^{\prime} S x=2 \sum_{i=1}^{k} \varepsilon_{i} x_{2 i-1} x_{2 i}+\sum_{i=2 k+1}^{n} \varepsilon_{i} x_{i}^{2}
$$

and

$$
G(x)=x^{\prime} T x=2 \lambda \sum_{i=1}^{k} \varepsilon_{i} x_{2 i-1} x_{2 i}+\sum_{i=1}^{k} \varepsilon_{i} x_{2 i}^{2}+\sum_{i=2 k+1}^{n} \varepsilon_{i} \mu_{i} x_{i}^{2},
$$

where $\varepsilon_{i}= \pm 1$. Thus $F(x)=G(x)=0$ is equivalent to $F(x)=0$ and

$$
\sum_{i=1}^{k} \varepsilon_{i} x_{2 i}^{2}+\sum_{i=2 k+1}^{n} \varepsilon_{i}\left(\mu_{i}-\lambda\right) x_{i}^{2}=0 .
$$

Assuming (vii) holds, then the quadratic form in (8) is indefinite, so there must exist an index $l$ such that

$$
\varepsilon_{1} \varepsilon_{l}\left(\mu_{l}-\lambda\right)<0 \quad \text { for some } l \geqq 2 k+1
$$

or such that

$$
\varepsilon_{1} \varepsilon_{l / 2}<0 \quad \text { for even } l \leqq 2 k \text {. }
$$

We define

$$
\begin{aligned}
& y_{1}=e_{1} \\
& y_{2}=\alpha_{2} e_{1}+\beta_{2} e_{2}+e_{l} \quad \text { for } \beta_{2} \neq 0, \\
& y_{l}=\alpha_{2} e_{1}+\beta_{2} e_{2}-e_{l}
\end{aligned}
$$

and

$$
y_{i}=\alpha_{i} e_{1}+\beta_{i} e_{2}+\gamma_{i} e_{l}+e_{i} \quad \text { for } i \neq 1,2, l, \beta_{i} \neq 0,
$$

where $\beta_{i}$ and $\gamma_{i}$ are chosen such that $y_{i}$ satisfies (8), while $\alpha_{i}$ are chosen such that $F\left(y_{i}\right)=0$. This proves (vii).

To prove (vii)(a) and (vii)(b) assume now that the quadratic form in (8) is semidefinite and that the symmetric matrix corresponding to the quadratic form in (8) has rank $n-k-r$, where the $r$ zeroes among the $\varepsilon_{i}\left(\mu_{i}-\lambda\right)$ occur for the indices $i=2 k+1, \cdots, 2 k+r$. Then the only unit vectors satisfying (8) are $e_{1}, e_{3}, \cdots, e_{2 k-1}, e_{2 k+1}, \cdots$, $e_{2 k+r}$. And clearly $e_{1}, e_{3}, \cdots, e_{2 k-1} \in Q_{S} \cap Q_{T}$ in either of the cases (vii)(a) 
or (vii)(b).

In case of (vii)(a) exactly $e_{1}, e_{3}, \cdots, e_{2 k-1} \in Q_{S} \cap Q_{T}$, because the quadratic form in $r$ variables

$$
\sum_{i=2 k+1}^{2 k+r} \varepsilon_{i} x_{i}^{2}
$$

appearing in $F$ is definite and $F(x) \neq 0$ for all $x$ with $x_{i} \neq 0$ for $2 k<$ $i \leqq 2 k+r$. So in this case we conclude that $Q_{S} \cap Q_{T}$ contains a maximum of $k$ lin. ind. vectors.

In case of (vii)(b)

$$
\sum_{i=2 k+1}^{2 k+r} \varepsilon_{i} x_{i}^{2}
$$

is an indefinite quadratic form and besides $e_{1}, e_{3}, \cdots, e_{2 k-1}, r$ more lin. indep. vectors $y_{1}, \cdots, y_{r}$ can be found that satisfy $F(x)=0$ and (8): Choose $y_{i}$ as follows. Since (9) is indefinite, there are indices $2 k<$ $l, j \leqq 2 k+r$ with $\varepsilon_{l}=1, \varepsilon_{j}=-1$. Then define for $2 k<i \leqq 2 k+r$, $i \neq l, j$ :

$$
y_{i}=e_{j}+e_{i} \quad \text { if } F\left(e_{i}\right)=1
$$

and

$$
y_{i}=e_{l}+e_{i} \quad \text { if } F\left(e_{i}\right)=-1
$$

while we set

$$
y_{l}=e_{j}+e_{l}
$$

and

$$
y_{j}=e_{j}-e_{l} \text {. }
$$

This proves (vii)(b).

(viii): Here we define

$$
F(x)=x^{\prime} S x=2 x_{1} x_{2}+\sum_{i=3}^{n} \varepsilon_{i} x_{i}^{2}
$$

and

$$
G(x)=x^{\prime} T x=2 a x_{1} x_{2}+b\left(x_{1}^{2}-x_{2}^{2}\right)+\sum_{i=3}^{n} \varepsilon_{i} \mu_{i} x_{i}^{2} .
$$

So $F(x)=G(x)=0$ is equivalent to

$$
F(x)=0 \quad \text { and } \quad b\left(x_{1}^{2}-x_{2}^{2}\right)+\sum_{i=3}^{n} \varepsilon_{i}\left(\mu_{i}-a\right) x_{i}^{2}=0 \text {. }
$$


Now unless (viii)(a) holds, not all $\mu_{i}$ or ${ }^{2}$ not all $\varepsilon_{i}$ are the same for $i \geqq 3$. So for some pair of indices $i, j \geqq 3$ we must have $\mu_{i} \neq$ $\mu_{j}$ or $^{2} \varepsilon_{i} \neq \varepsilon_{j}$. After a suitable index permutation we may start the proof assuming that $\mu_{3} \neq \mu_{4}$ or $^{2} \varepsilon_{3} \neq \varepsilon_{4}$ already.

We define $y_{1}=\alpha_{3} e_{1}+\beta_{3} e_{2}-e_{3}, y_{2}=\alpha_{4} e_{1}+\beta_{4} e_{2}-e_{4}$ and $y_{i}=\alpha_{i} e_{1}+$ $\beta_{i} e_{2}+e_{i}$ for $i \geqq 3$, where the $\alpha_{i}, \beta_{i}$ are chosen to satisfy

(11) $b\left(\alpha_{i}^{2}-\beta_{i}^{2}\right)+\varepsilon_{i}\left(\mu_{i}-\alpha\right)=0$ and $2 \alpha_{i} \beta_{i}+\varepsilon_{i}=0$ for each $i$.

Then the vectors $y_{i}$ for $i \leqq n$ are lin. ind. iff

$$
\operatorname{det}\left(y_{1}, \cdots, y_{n}\right)=\left|\begin{array}{rrrr}
\alpha_{3} & \beta_{3} & -1 & 0 \\
\alpha_{4} & \beta_{4} & 0 & -0 \\
\alpha_{3} & \beta_{3} & 1 & 0 \\
\alpha_{4} & \beta_{4} & 0 & 1
\end{array}\right|=\left|\begin{array}{cccc}
2 \alpha_{3} & 2 \beta_{3} & 0 & 0 \\
2 \alpha_{4} & 2 \beta_{4} & 0 & 0 \\
\alpha_{3} & \beta_{3} & 1 & 0 \\
\alpha_{4} & \beta_{4} & 0 & 1
\end{array}\right| \neq 0 \text {. }
$$

So the $n$ vectors $y_{i}$ are lin. dep. iff for the 2 -vectors we have

$$
\left(\alpha_{3}, \beta_{3}\right)=d\left(\alpha_{4}, \beta_{4}\right)
$$

for some real coefficient $d$. Now (12) holds only if $d= \pm 1$, for (12) implies $\alpha_{3} \beta_{3}=d^{2} \alpha_{4} \beta_{4}$ and we know $\alpha_{3} \beta_{3}=-\varepsilon_{3} / 2$, since $F^{\prime}\left(y_{3}\right)=0$ and $\alpha_{4} \beta_{4}=-\varepsilon 4 / 2$, since $F\left(y_{4}\right)=0$ and thus $d= \pm 1$.

If $d=1$, then by (11) we have $\varepsilon_{3}=\varepsilon_{4}$ and hence by assumption $\mu_{3} \neq \mu_{4}$ which contradicts (11). If $d=-1$, then $\varepsilon_{3}=-\varepsilon_{4}$ and $\alpha_{3} \beta_{3}=-$ $\alpha_{4} \beta_{4}$ by (11), contradicting (12).

Thus we found that the $n$ vectors $y_{i}$ in $Q_{S} \cap Q_{T}$ are lin. ind. in case of (viii).

If (viii)(a) holds, $\mu_{i}=\mu$ and $\varepsilon_{i}=\varepsilon$ for all $i$. We define for $x=$ $\left(x_{1}, \cdots, x_{n}\right)$

$$
F(x)=x^{\prime} S x=2 x_{1} x_{2}+\varepsilon \sum_{i=3}^{n} x_{i}^{2}
$$

and

$$
G(x)=x^{\prime} T x=2 a x_{1} x_{2}+b\left(x_{1}^{2}-x_{2}^{2}\right)+\varepsilon \mu \sum_{i=3}^{n} x_{i}^{2} .
$$

And $F(x)=G(x)=0$ is equivalent to

$$
F(x)=0 \quad \text { and } \quad b\left(x_{1}^{2}-x_{2}^{2}\right)+\varepsilon(\mu-a) \sum_{i=3}^{n} x_{i}^{2}=0 .
$$

We define the following $n-1$ lin. ind. vectors

$$
\begin{aligned}
& y_{i}=\alpha e_{1}+\beta e_{2}+e_{i} \quad \text { for } i \geqq 3, \\
& y_{2}=\alpha e_{1}+\beta e_{2}-e_{3},
\end{aligned}
$$

2 This "or" does not mean "either... or". 
where $\alpha, \beta$ are chosen such that $F\left(y_{i}\right)=G\left(y_{i}\right)=0$ for all $i$. Such numbers $\alpha, \beta$ exist, since they can be chosen as the intersection of the following two hyperbolas

$$
2 \alpha \beta+\varepsilon=0 ; \quad \alpha^{2}-\beta^{2}=-\frac{\varepsilon}{b}(\mu-a) .
$$

Now any $w=\left(\beta_{1}, \cdots, \beta_{n}\right) \in Q_{S} \cap Q_{T}$ satisfies (14). We are going to show that if $0 \neq w \in Q_{S} \cap Q_{T}$ then the 2-vector $\left(\beta_{1}, \beta_{2}\right)$ can be written as $\pm\|\hat{x}\|(\alpha, \beta)$ with $\alpha, \beta$ as chosen above and $\hat{x}=\left(0,0, \beta_{3}, \cdots, \beta_{n}\right)$. Now if $\|\widehat{x}\|=0$, i.e., $\beta_{i}=0$ for all $i \geqq 3$, then by (14) $\beta_{1} \beta_{2}=0=$ $\beta_{1}^{2}-\beta_{2}^{2}$ so that $w=0$. If $\beta_{1}=0$, then by (13) we get $w=0$.

So if $w \neq 0$ belongs to $Q_{S} \cap Q_{T}$, then $\|\hat{x}\| \neq 0$ and we define $d$ as $d=\beta_{1} / \alpha$ with $\alpha$ as introduced above. Using the equations $F(w)=$ $F\left(y_{i}\right)=0$ we get $2 \alpha \beta=-\varepsilon=2 \beta_{1} \beta_{2} /\|\hat{x}\|^{2}$ and hence $\beta_{2}=\|\hat{x}\| \|^{2} \beta / d$. The second equation in (14), written out for $y_{i}$ and $w$, reads like

$$
b\left(\alpha^{2}-\beta^{2}\right)+\varepsilon(\mu-a)=0=b\left(d^{2} \alpha^{2}-\|\hat{x}\|^{4} \beta^{2} / d^{2}\right)+\varepsilon(\mu-a)\|\hat{x}\|^{2},
$$

and hence

$$
\alpha^{2}-\beta^{2}=d^{2} \alpha^{2} /\|\hat{x}\|^{2}-\|\hat{x}\|^{2} \beta^{2} / d^{2}
$$

or

$$
d^{4} \alpha^{2}+d^{2}\|\hat{x}\|^{2}\left(\beta^{2}-\alpha^{2}\right)-\beta^{2}\|\hat{x}\|^{4}=0 .
$$

This last equation in $d$ has only two real roots, namely $d= \pm\|\hat{x}\|$. Hence $\beta_{2}= \pm\|\hat{x}\| \beta$, while $\beta_{1}= \pm\|\hat{x}\| \alpha$.

So the equation $w=\left(\beta_{1}, \cdots, \beta_{n}\right)=d\left(\alpha e_{1}+\beta e_{2}\right)+\left(0,0, \beta_{3}, \cdots\right.$, $\left.\beta_{n}\right)=\sum_{i=2}^{n} \eta_{i} y_{i}$ can be solved for real coefficients $\eta_{i}$, namely by $\eta_{i}=$ $\beta_{i}$ for $i>3$,

$$
\eta_{2}=\left(d-\sum_{i=3}^{n} \beta_{i}\right) / 2
$$

and $\eta_{3}=\beta_{3}+\eta_{2}$, where

$$
d= \pm\left(\sum_{i=3}^{n} \beta_{i}^{2}\right)^{1 / 2}
$$

as we have seen above.

So every $w \in Q_{S} \cap Q_{T}$ is lin. dep. of $y_{2}, \cdots, y_{n}$ and in this case $n-1$ is the maximal number of lin. ind. vectors in $Q_{S} \cap Q_{T}$. This proves (viii)(a).

(ix): It only remains to show (ix): Let $S$ and $T$ be simultaneously diagonalizable.

Assume $S$ is positive definite, then $Q_{S}=\{0\}$ and hence for any symmetric $T$ we have $Q_{S} \cap Q_{T}=\{0\}$, hence the case $k=0$ occurs. 
If $S=\operatorname{diag}(1,-1, \cdots,-1,1, \cdots, 1)$ and $T=\operatorname{diag}(\lambda,-\lambda, \cdots$, $-\lambda, 0, \cdots, 0)$ with $(l-1)$ numbers -1 and $-\lambda$ appearing on the diagonals of $S$ and $T$, then $Q_{S} \cap Q_{T}$ contains a maximum of $l$ lin. indep. vectors for $\lambda \neq 0,2 \leqq l \leqq n$ as can be seen by inspection. Finally if $x \in Q_{S} \cap Q_{T}$, then $x$ can be written as $x=\alpha e_{l}+\beta e_{k}+y$ for two indices $l, k$, nonzero constants $\alpha, \beta$, and $y$ orthogonal to $e_{l}$ and $e_{k}$, because $x$ has to satisfy

$$
F(x)=x^{\prime} S x=\sum_{i=1}^{n} \varepsilon_{i} x_{i}^{2}=0
$$

and

$$
G(x)=x^{\prime} T x=\sum_{i=1}^{n} \varepsilon_{i} \mu_{i} x_{i}^{2}=0, \quad \text { with } \varepsilon_{i}= \pm 1 .
$$

But then $\hat{x}=\alpha e_{l}-\beta e_{k}+y \in Q_{S} \cap Q_{T}$ as well and $x$ and $\hat{x}$ are lin. ind. So in case (ix) $Q_{S} \cap Q_{T}$ cannot contain just one vector and its multiples.

This proves Theorem 2.

Next we treat nonsingular pairs of real symmetric matrices that have dimensions 2 or 3 .

THeOREM 3. Let $S, T$ be a nonsingular pair of r.s. matrices of dimension n. Assume that $n=2$ or 3 . Let the Roman numerals (vi), ..., (viii) denote the various cases of Theorem 2.

If (vii) holds, then $Q_{S} \cap Q_{T}$ contains $n$ lin. ind. vectors.

In (vi)(a) or (viii)(a) (with $n=3$ ) holds, then $Q_{S} \cap T_{T}$ contains a maximum of $n-1$ lin. ind. vectors.

If (vii)(a) holds, then $Q_{S} \cap Q_{T}$ contains a maximum of $k$ lin. ind. vectors, where $k$ is defined as in Theorem 2.

If (viii)(a) holds with $n=2$, then $Q_{S} \cap Q_{T}=\{0\}$.

Proof. In view of Lemma 2 we can again assume that $S$ and $T$ are already in canonical pair form.

(a) Let $n=3$ : If $J=S^{-1} T$ contains just one 3-dimensional block $J(\lambda, 3)$, then inertia $S=(2,1,0)$ or $(1,2,0)$ and we have condition (vi)(a). Then $F(x)=x^{\prime} S x=\varepsilon\left(2 x_{1} x_{3}+x_{2}^{2}\right)$ and $G(x)=x^{\prime} T x=\varepsilon\left(\lambda\left(2 x_{1} x_{3}+\right.\right.$ $\left.x_{2}^{2}\right)+2 x_{2} x_{3}$ ) with $\varepsilon= \pm 1$. Hence the only vectors $x$ satisfying $F(x)=$ $G(x)=0$ are multiples of $e_{1}$ and of $e_{3}$. Hence there are at most 2 lin. ind. vectors in $Q_{S} \cap Q_{T}$.

If $S^{-1} T$ has a complex root, then we have case (viii)(a) and the proof of Theorem 2 (viii)(a) carries over. 
If $S^{-1} T$ satisfies condition (vii), then we have for $\varepsilon_{i}= \pm 1, F(x)=$ $x^{\prime} S x=\varepsilon_{1}\left(2 x_{1} x_{2}\right)+\varepsilon_{3} x_{3}^{2}$ and $G(X)=x^{\prime} T x=\varepsilon_{1}\left(\lambda 2 x_{1} x_{2}+x_{2}^{2}\right)+\varepsilon_{3} \mu x_{3}^{2}$ and thus $F(x)=G(x)=0$ is equivalent to

$$
F(x)=0 \text { and } \varepsilon_{1} x_{2}^{2}+\varepsilon_{3}(\mu-\lambda) x_{3}^{2}=0 .
$$

If $\lambda=\mu$, then only multiples of $e_{1}$ are in $Q_{S} \cap Q_{T}$ and if $\lambda \neq \mu$, but $\varepsilon_{1} \varepsilon_{3}(\mu-\lambda)>0$, then again only multiples of $e_{1}$ are in $Q_{S} \cap Q_{T}$. Now condition (vii)(a) encompasses exactly these two cases, hence if (vii)(a) holds, then $Q_{S} \cap Q_{T}$ is just a one dimensional space.

If (vii) holds, i.e., $\lambda \neq \mu$ and $\varepsilon_{1} \varepsilon_{3}(\mu-\lambda)<0$, then we define

$$
\begin{aligned}
& y_{1}=e_{1} \\
& y_{2}=\alpha e_{1}+\beta e_{2}-e_{3}
\end{aligned}
$$

and

$$
y_{3}=\alpha e_{1}+\beta e_{2}+e_{3} \quad \text { where } \alpha, \beta \neq 0
$$

are such that $y_{2}, y_{3}$ satisfy (15).

(b) If $n=2$, we have in case of just one Jordan block $J(\lambda, 2)$ in $J=S^{-1} T: F(x)=x^{\prime} S x=\varepsilon\left(2 x_{1} x_{2}\right)$ and $G(x)=x^{\prime} T x=\varepsilon\left(2 \lambda x_{1} x_{2}+x_{2}^{2}\right)$ for $\varepsilon= \pm 1$. So $F(x)=G(x)=0$ holds iff $x=\alpha e_{1}$. Hence (vii)(a) is proved. In case of (viii)(a) for a Jordan block $J(a, b, 2)$ of type (B), we have $F(x)=2 x_{1} x_{2}$ and $G(x)+2 a x_{1} x_{2}+b\left(x_{1}^{2}-x_{2}^{2}\right)$. And hence $F(x)=$ $G(x)=0$ holds iff $x=0$.

Let $S$ and $T$ be a nonsingular pair of r.s. matrices of dimension greater than 2. In Theorems 1, 2, and 3 we have seen how the real Jordan normal form of $S^{-1} T$ determines the maximal number of lin. ind. vectors in $Q_{S} \cap Q_{T}$. Since we have dealt with all possible real Jordan normal forms, we can reverse the argument and get the following:

THEOREM 4. Let $S$ and $T$ be a nonsingular pair of r.s. $n \times n$ matrices where $n>2$.

Let $m=\max \left\{l \mid\right.$ there exist $l$ lin. ind. vectors in $\left.Q_{S} \cap Q_{T}\right\}$. Let the Roman numerals (i), $\cdots$, (viii) denote the various conditions in Theorem 2.

If $m=0$, then $S$ and $T$ can be simultaneously diagonalized by a real congruence transformation.

If $m=1$, then (vii)(a) holds with $k=1$.

If $2 \leqq m \leqq[n / 2]$, then (vii)(a) holds with $k=m$ or (vii)(b) holds with $r=m-k$ for $S$ and $T$, or $S$ and $T$ can be diagonalized simultaneously. 
If $[n / 2]<m<n-1$, then (vii)(b) holds with $r=m-k$ where $k \leqq[n / 2]$ for $S$ and $T$, or $S$ and $T$ can be diagonalized simultaneously.

If $m=n-1$, then (vi)(a) or (viii)(a) or (vii)(b) holds with $r=$ $m-k$, where $k \leqq[n / 2]$ for $S$ and $T$, or $S$ and $T$ can be diagonalized simultaneously.

If $m=n$, then (i), $\cdots$ or (viii) holds for $S$ and $T$, or $S$ and $T$ can be diagonalized simultaneously by a real congruence transformation.

Here [ 1 denotes the greatest integer function.

If $m$, the maximal number of lin. ind. vectors simultaneously annihilated by two quadratic forms $x^{\prime} S x, x^{\prime} T x$, lies properly between 1 and $n-1$, and if we can rule out the cases (vii)(a) or (vii)(b), then we can conclude that $S$ and $T$ are simultaneously diagonalizable. For example, here are two such conditions that make (vii)(a) or (vii)(b) impossible to happen:

COROLlaRY 1. Let $S$ and $T$ be a nonsingular part of r.s. $n \times n$ matrices. Let $m=\max \left\{l \mid\right.$ there exist $l$ lin. ind. vectors in $\left.Q_{S} \cap Q_{T}\right\}$. Assume $1<m<n-1$.

If (a) $S^{-1} T$ is nonderogatory, or

(b) for every eigenvalue $\lambda$ of $S^{-1} T$ the number of associated lin. ind. eigenvectors is smaller than half the algebraic multiplicity of $\lambda$, unless both are the same, then $S$ and $T$ can be diagonalized simultaneously by a real congruence transformation.

Nonderogators matrices are those that have only one Jordan block for each different eigenvalue.

As a further corollary to Theorem $4(m=0)$ we get a result due to Greub and Milnor [1, p. 256]:

COROLLARY 2. Let $S$ and $T$ be a nonsingular pair of r.s. matrices. If $Q_{S} \cap Q_{T}=\{0\}$, then $S$ and $T$ can be diagonalized simultaneously be a real congruence transformation.

ACKNowledgment. This paper in essence constitutes Chapter 4 of my Ph. D. Thesis at the California Institute of Technology, 1971. I am indebted to my advisor Dr. Olga Taussky-Todd and to Dr. H. F. Bohnenblust for their guidance and advice. Dr. Olga Taussky-Todd suggested that in order to generalize the theorems known about simultaneous diagonalization of symmetric matrices one ought to study the set $Q_{S} \cap Q_{T}$. 


\section{REFERENCES}

1. W. Greub, Linear Algebra, Springer, Berlin, 3 $3^{\text {rd }}$ edition, 1967.

2. H. J. Kowalski, Lineare Algebra, Göschens Lehrbücherei 27, Berlin, 1963.

3. F. Uhlig, Simultaneous block diagonalization of two real symmetric matrices, Linear Algebra and Appl., 7 (1973), 281-289.

Received July 12, 1972.

UNIVERSITÄT WÜRZBURG 


\section{PACIFIC JOURNAL OF MATHEMATICS}

\section{EDITORS}

RICHARD ARENS (Managing Editor)

University of California

Los Angeles, California 90024

\section{R. A. Beaumont}

University of Washington

Seattle, Washington 98105

\section{J. DuGundJI*}

Department of Mathematics

University of Southern California

Los Angeles, California 90007

D. Gilbarg and J. Milgram

Stanford University

Stanford, California 94305

ASSOCIATE EDITORS
E. F. BECKENBACH
B. H. NeumanN
F. WOLF
K. YoSHIDA

\section{SUPPORTING INSTITUTIONS}

UNIVERSITY OF BRITISH COLUMBIA

CALIFORNIA INSTITUTE OF TECHNOLOGY

UNIVERSITY OF CALIFORNIA

MONTANA STATE UNIVERSITY

UNIVERSITY OF NEVADA

NEW MEXICO STATE UNIVERSITY

OREGON STATE UNIVERSITY

UNIVERSITY OF OREGON

OSAKA UNIVERSITY

\author{
UNIVERSITY OF SOUTHERN CALIFORNIA \\ STANFORD UNIVERSITY \\ UNIVERSITY OF TOKYO \\ UNIVERSITY OF UTAH \\ WASHINGTON STATE UNIVERSITY \\ UNIVERSITY OF WASHINGTON \\ AMERICAN MATHEMATICAL SOCIETY \\ NAVAL WEAPONS CENTER
}

The Supporting Institutions listed above contribute to the cost of publication of this Journal, but they are not owners or publishers and have no responsibility for its content or policies.

Mathematical papers intended for publication in the Pacific Journal of Mathematics should be in typed form or offset-reproduced, (not dittoed), double spaced with large margins. Underline Greek letters in red, German in green, and script in blue. The first paragraph or two must be capable of being used separately as a synopsis of the entire paper. Items of the bibliography should not be cited there unless absolutely necessary, in which case they must be identified by author and Journal, rather than by item number. Manuscripts, in duplicate if possible, may be sent to any one of the four editors. Please classify according to the scheme of Math. Rev. Index to Vol. 39. All other communications to the editors should be addressed to the managing editor, or Elaine Barth, University of California, Los Angeles, California, 90024.

50 reprints are provided free for each article; additional copies may be obtained at cost in multiples of 50 .

The Pacific Journal of Mathematics is issued monthly as of January 1966. Regular subscription rate: $\$ 48.00$ a year (6 Vols., 12 issues). Special rate: $\$ 24.00$ a year to individual members of supporting institutions.

Subscriptions, orders for back numbers, and changes of address should be sent to Pacific Journal of Mathematics, 103 Highland Boulevard, Berkeley, California, 94708.

\section{PUBLISHED BY PACIFIC JOURNAL OF MATHEMATICS, A NON-PROFIT CORPORATION}

Printed at Kokusai Bunken Insatsusha (International Academic Printing Co., Ltd.), 270, 3-chome Totsuka-cho, Shinjuku-ku, Tokyo 160, Japan.

* C. R. DePrima California Institute of Technology, Pasadena, CA 91109, will replace J. Dugundji until August 1974.

Copyright (C) 1973 by

Pacific Journal of Mathematics

All Rights Reserved 


\section{Pacific Journal of Mathematics}

\section{Vol. 49, No. $2 \quad$ June, 1973}

Wm. R. Allaway, On finding the distribution function for an orthogonal polynomial

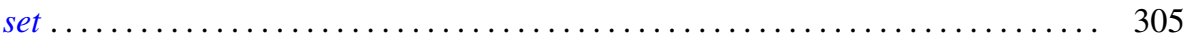

Eric Amar, Sur un théorème de Mooney relatif aux fonctions analytiques bornées... . 311

Robert Morgan Brooks, Analytic structure in the spectrum of a natural system . . . . 315

Bahattin Cengiz, On extremely regular function spaces . . . . . . . . . . . . . . 335

Kwang-nan Chow and Moses Glasner, Atoms on the Royden boundary . . . . . . . . . 339

Paul Frazier Duvall, Jr. and Jim Maxwell, Tame $Z^{2}$-actions on $E^{n} \ldots \ldots \ldots \ldots \ldots . .349$

Allen Roy Freedman, On the additivity theorem for $n$-dimensional asymptotic

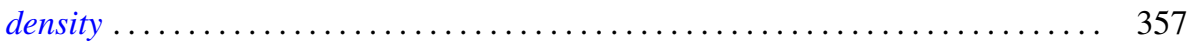

John Griffin and Kelly Denis McKennon, Multipliers and the group $L_{p}$-algebras . . . 365

Charles Lemuel Hagopian, Characterizations of $\lambda$ connected plane continua ....... 371

Jon Craig Helton, Bounds for products of interval functions . . . . . . . . . . . 377

Ikuko Kayashima, On relations between Nörlund and Riesz means . . . . . . . . . . 391

Everett Lee Lady, Slender rings and modules . . . . . . . . . . . . . . . . . . 397

Shozo Matsuura, On the Lu Qi-Keng conjecture and the Bergman representative

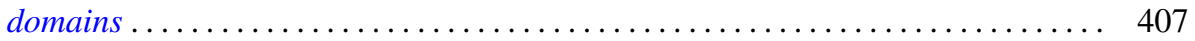

Stephen H. McCleary, The lattice-ordered group of automorphisms of an $\alpha$-set . . . 417

Stephen H. McCleary, o-2-transitive ordered permutation groups .......... 425

Stephen H. McCleary, o-primitive ordered permutation groups. II . . . . . . . . . 431

Richard Rochberg, Almost isometries of Banach spaces and moduli of planar

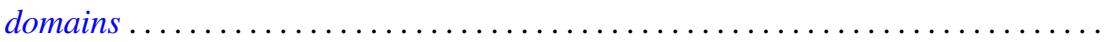

R. F. Rossa, Radical properties involving one-sided ideals . . . . . . . . . . . . . 467

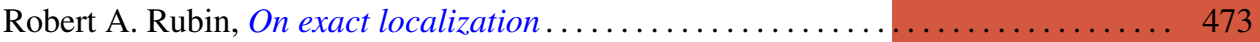

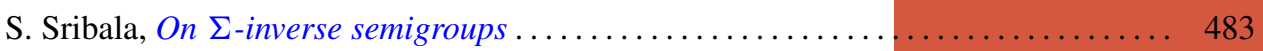

H. M. (Hari Mohan) Srivastava, On the Konhauser sets of biorthogonal polynomials suggested by the Laguerre polynomials ...................... 489

Stuart A. Steinberg, Rings of quotients of rings without nilpotent elements . ...... 493

Daniel Mullane Sunday, The self-equivalences of an $H$-space . . ............ 507

W. J. Thron and Richard Hawks Warren, On the lattice of proximities of $\check{C} e c h$

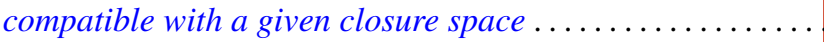

Frank Uhlig, The number of vectors jointly annihilated by two real quadratic forms determines the inertia of matrices in the associated pencil .

Frank Uhlig, On the maximal number of linearly independent real vectors annihilated simultaneously by two real quadratic forms ..............

Frank Uhlig, Definite and semidefinite matrices in a real symmetric matrix pencil . . 561

Arnold Lewis Villone, Self-adjoint extensions of symmetric differential operators . . . 569

Cary Webb, Tensor and direct products . ....................... 579

James Victor Whittaker, On normal subgroups of differentiable

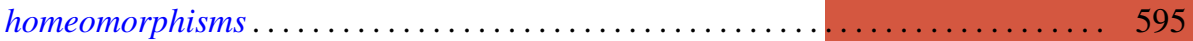

Jerome L. Paul, Addendum to: "Sequences of homeomorphisms which converge to

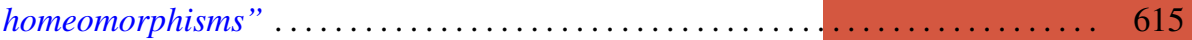

David E. Fields, Correction to: “Dimension theory in power series rings” ........ 616

Peter Michael Curran, Correction to: “Cohomology of finitely presented groups”. . . 617

Billy E. Rhoades, Correction to: “Commutants of some Hausdorff matrices” ...... 617

Charles W. Trigg, Corrections to: "Versum sequences in the binary system” ...... 619 